\title{
On the binomial edge ideals of block graphs
}

\author{
Faryal Chaudhry, Ahmet Dokuyucu, Rida Irfan
}

\begin{abstract}
We find a class of block graphs whose binomial edge ideals have minimal regularity. As a consequence, we characterize the trees whose binomial edge ideals have minimal regularity. Also, we show that the binomial edge ideal of a block graph has the same depth as its initial ideal.
\end{abstract}

\section{Introduction}

In this paper we study homological properties of some classes of binomial edge ideals.

Let $G$ be a simple graph on the vertex set $[n]$ and let $S=K\left[x_{1}, \ldots, x_{n}, y_{1}, \ldots, y_{n}\right]$ be the polynomial ring in $2 n$ variables over a field $K$. For $1 \leq i<j \leq n$, we set $f_{i j}=x_{i} y_{j}-x_{j} y_{i}$. The binomial edge ideal of $G$ is defined as $J_{G}=\left(f_{i j}:\{i, j\} \in E(G)\right)$. Binomial edge ideals were introduced in [8] and [12]. Algebraic and homological properties of binomial edge ideals have been studied in several papers. In [5], it was conjectured that $J_{G}$ and in $_{<}\left(J_{G}\right)$ have the same extremal Betti numbers. Here $<$ denotes the lexicographic order in $S$ induced by $x_{1}>x_{2}>\cdots>x_{n}>y_{1}>y_{2}>\cdots>y_{n}$. This conjecture was proved in [3] for cycles and complete bipartite graphs. In [6], it was shown that, for a closed graph $G, J_{G}$ and $\operatorname{in}_{<}\left(J_{G}\right)$ have the same regularity which can be expressed in the combinatorial data of the graph. We recall that a graph $G$ is closed if and only if it has a quadratic Gröbner basis with respect to the lexicographic order.

Key Words: Binomial edge ideals, regularity, depth.

2010 Mathematics Subject Classification: 13D02, 05E40.

Received: 04.02.2015

Accepted: 02.03.2015 
In support of the conjecture given in [5], we show, in Section 3, that if $G$ is a block graph, then $\operatorname{depth}\left(S / J_{G}\right)=\operatorname{depth}\left(S / \operatorname{in}_{<}\left(J_{G}\right)\right)$; see Theorem 3.2. By a block graph we mean a chordal graph $G$ with the property that any two maximal cliques of $G$ intersect in at most one vertex.

Also, in the same section, we show a similar equality for regularity. More precisely, in Theorem 3.4 we show that $\operatorname{reg}\left(S / J_{G}\right)=\operatorname{reg}\left(S / \mathrm{in}_{<}\left(J_{G}\right)\right)=\ell$ if $G$

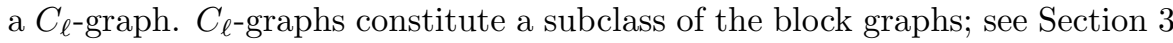
for definition and Figure 1 for an example.

In [10] it was shown that, for any connected graph $G$ on the vertex set $[n]$, we have

$$
\ell \leq \operatorname{reg}\left(S / J_{G}\right) \leq n-1,
$$

where $\ell$ is the length of the longest induced path of $G$.

The main motivation of our work was to answer the following question. May we characterize the connected graphs $G$ whose longest induced path has length $\ell$ and $\operatorname{reg}\left(S / J_{G}\right)=\ell$ ? We succeeded to answer this question for trees. In Theorem 4.1, we show that if $T$ is a tree whose longest induced path has length $\ell$, then $\operatorname{reg}\left(S / J_{T}\right)=\ell$ if and only if $T$ is caterpillar. A caterpillar tree is a tree $T$ with the property that it contains a path $P$ such that any vertex of $T$ is either a vertex of $P$ or it is adjacent to a vertex of $P$.

In [11], the so-called weakly closed graphs were introduced. This is a class of graphs which includes closed graphs. In the same paper, it was shown that a tree is caterpillar if and only if it is a weakly closed graph. Having in mind our Theorem 4.1 and Theorem 3.2 in [6] which states that $\operatorname{reg}\left(S / J_{G}\right)=\ell$ if $G$ is a connected closed graph whose longest induced path has length $\ell$, and by some computer experiments, we are tempted to formulate the following.

Conjecture 1.1. If $G$ is a connected weakly closed graph whose longest induced path has length $\ell$, then $\operatorname{reg}\left(S / J_{G}\right)=\ell$.

\section{Preliminaries}

In this section we introduce the notation used in this paper and summarize a few results on binomial edge ideals.

Let $G$ be a simple graph on the vertex set $[n]=\{1, \ldots, n\}$, that is, $G$ has no loops and no multiple edges. Furthermore, let $K$ be a field and $S=$ $K\left[x_{1}, \ldots, x_{n}, y_{1}, \ldots, y_{n}\right]$ be the polynomial ring in $2 n$ variables. For $1 \leq i<$ $j \leq n$, we set $f_{i j}=x_{i} y_{j}-x_{j} y_{i}$. The binomial edge ideal $J_{G} \subset S$ associated with $G$ is generated by all the quadratic binomials $f_{i j}=x_{i} y_{j}-x_{j} y_{i}$ such that $\{i, j\} \in E(G)$. Binomial edge ideals were introduced in the papers [8] and [12].

We first recall some basic definitions from graph theory. A vertex $i$ of $G$ whose deletion from the graph gives a graph with more connected components 
than $G$ is called a cut point of G. A chordal graph is a graph without cycles of length greater than or equal to 4 . A clique of a graph $G$ is a complete subgraph of $G$. The cliques of a graph $G$ form a simplicial complex, $\Delta(G)$, which is called the clique complex of $G$. Its facets are the maximal cliques of $G$. A graph $G$ is a block graph if and only if it is chordal and every two maximal cliques have at most one vertex in common. This class was considered in [5, Theorem 1.1].

The clique complex $\Delta(G)$ of a chordal graph $G$ has the property that there exists a leaf order on its facets. This means that the facets of $\Delta(G)$ may be ordered as $F_{1}, \ldots, F_{r}$ such that, for every $i>1, F_{i}$ is a leaf of the simplicial complex generated by $F_{1}, \ldots, F_{i}$. A leaf $F$ of a simplicial complex $\Delta$ is a facet of $\Delta$ with the property that there exists another facet of $\Delta$, say $G$, such that, for every facet $H \neq F$ of $\Delta, H \cap F \subseteq G \cap F$.

Let $<$ be the lexicographic order on $S$ induced by the natural order of the variables. As it was shown in [8, Theorem 2.1], the Gröbner basis of $J_{G}$ with respect to this order may be given in terms of the admissible paths of $G$. We recall the definition of admissible paths from [8].

Definition 2.1. [8] Let $i<j$ be two vertices of $G$. A path $i=i_{0}, i_{1}, \ldots, i_{r-1}, i_{r}=j$ from $i$ to $j$ is called admissible if the following conditions are fulfilled:

1. $i_{k} \neq i_{l}$ for $k \neq l$;

2. for each $k=1, \ldots, r-1$ on has either $i_{k}<i$ or $i_{k}>j$;

3. for any proper subset $\left\{j_{1}, \ldots, j_{s}\right\}$ of $\left\{i_{1}, \ldots, i_{r-1}\right\}$, the sequence $i, j_{1}, \ldots, j_{s}, j$ is not a path in $G$.

Given an admissible path $\pi$ in $G$ from $i$ to $j$, we set $u_{\pi}=\left(\prod_{i_{k}>j} x_{i_{k}}\right)\left(\prod_{i_{l}<i} y_{i_{l}}\right)$.

By [8, Theorem 2.1], it follows that

$$
\operatorname{in}_{<}\left(J_{G}\right)=\left(u_{\pi} x_{i} y_{j}: i<j, \pi \text { is an admissible path from } i \text { to } j\right) .
$$

In particular, $\operatorname{in}_{<}\left(J_{G}\right)$ is a radical monomial ideal which implies that the binomial edge ideal $J_{G}$ is radical as well. Hence $J_{G}$ is equal to the intersection of all its minimal prime ideals. The minimal prime ideals were determined in $[8$, Section 3] in terms of the combinatorial data of the graph.

\section{Initial ideals of binomial edge ideals of block graphs}

In this section, we first show that, for a block graph $G$ on $[n]$ with $c$ connected components, we have $\operatorname{depth}\left(S / J_{G}\right)=\operatorname{depth}\left(S / \operatorname{in}_{<}\left(J_{G}\right)\right)=n+c$, where $<$ 
denotes the lexicographic order induced by $x_{1}>\cdots>x_{n}>y_{1}>\cdots>y_{n}$ in the ring $S=K\left[x_{1}, \ldots, x_{n}, y_{1}, \ldots, y_{n}\right]$.

We begin with the following lemma.

Lemma 3.1. Let $G$ be a graph on the vertex set $[n]$ and let $i \in[n]$. Then

$$
\operatorname{in}_{<}\left(J_{G}, x_{i}, y_{i}\right)=\left(\operatorname{in}_{<}\left(J_{G}\right), x_{i}, y_{i}\right) .
$$

Proof. We have $\operatorname{in}_{<}\left(J_{G}, x_{i}, y_{i}\right)=\operatorname{in}_{<}\left(J_{G \backslash\{i\}}, x_{i}, y_{i}\right)=\left(\operatorname{in}_{<}\left(J_{G \backslash\{i\}}\right), x_{i}, y_{i}\right)$. Therefore, we have to show that $\left(\operatorname{in}_{<}\left(J_{G}\right), x_{i}, y_{i}\right)=\left(\operatorname{in}_{<}\left(J_{G \backslash\{i\}}\right), x_{i}, y_{i}\right)$. The inclusion $\supseteq$ is obvious since $J_{G \backslash\{i\}} \subset J_{G}$. For the other inclusion, let us take $u$ to be a minimal generator of $\operatorname{in}_{<}\left(J_{G}\right)$. If $x_{i} \mid u$ or $y_{i} \mid u$, obviously $u \in\left(\operatorname{in}\left(J_{G \backslash\{i\}}\right), x_{i}, y_{i}\right)$. Let now $x_{i} \nmid u$ and $y_{i} \nmid u$. This means that $u=u_{\pi} x_{k} y_{l}$ for some admissible path $\pi$ from $k$ to $l$ which does not contain the vertex $i$. Then it follows that $\pi$ is a path from $k$ to $l$ in $G \backslash\{i\}$, hence $u \in \mathrm{in}_{<}\left(J_{G \backslash\{i\}}\right)$.

Theorem 3.2. Let $G$ be a block graph. Then

$$
\operatorname{depth}\left(S / J_{G}\right)=\operatorname{depth}\left(S / \operatorname{in}_{<}\left(J_{G}\right)\right)=n+c,
$$

where $c$ is the number of connected component of $G$.

Proof. Let $G_{1}, \ldots, G_{c}$ be the connected components of $G$ and $S_{i}=K\left[\left\{x_{j}, y_{j}\right\}_{j \in G_{i}}\right]$. Then $S / J_{G} \cong S_{1} / J_{G_{1}} \otimes \cdots \otimes S_{c} / J_{G_{c}}$, so that

$$
\operatorname{depth} S / J_{G}=\operatorname{depth} S_{1} / J_{G_{1}}+\cdots+\operatorname{depth} S_{c} / J_{G_{c}} .
$$

Moreover, we have $S /$ in $_{<}\left(J_{G}\right) \cong S /$ in $_{<}\left(J_{G_{1}}\right) \otimes \cdots \otimes S /$ in $_{<}\left(J_{G_{c}}\right)$, thus

$$
\operatorname{depth} S / \operatorname{in}_{<}\left(J_{G}\right)=\operatorname{depth} S_{1} / \operatorname{in}_{<}\left(J_{G_{1}}\right)+\cdots+\operatorname{depth} S_{c} / \operatorname{in}_{<}\left(J_{G_{c}}\right) .
$$

Hence, without loss of generality, we may assume that $G$ is connected. By [5, Theorem 1.1] we know that $\operatorname{depth}\left(S / J_{G}\right)=n+1$. In order to show that $\operatorname{depth}\left(S /\right.$ in $\left._{<}\left(J_{G}\right)\right)=n+1$, we proceed by induction on the number of maximal cliques of $G$. Let $\Delta(G)$ be the clique complex of $G$ and let $F_{1}, \ldots, F_{r}$ be a leaf order on the facets of $\Delta(G)$. If $r=1$, then $G$ is a simplex and the statement is well known. Let $r>1$; since $F_{r}$ is a leaf, there exists a unique vertex, say $i \in F_{r}$, such that $F_{r} \cap F_{j}=\{i\}$ where $F_{j}$ is a branch of $F_{r}$. Let $F_{t_{1}}, \ldots, F_{t_{q}}$ be the facets of $\Delta(G)$ which intersect the leaf $F_{r}$ in the vertex $\{i\}$. Following the proof of [5, Theorem 1.1] we may write $J_{G}=J_{1} \cap J_{2}$ where $J_{1}=\bigcap_{i \notin \mathcal{S}} P_{\mathcal{S}}(G)$ and $J_{2}=\bigcap_{i \in \mathcal{S}} P_{\mathcal{S}}(G)$. Then, as it was shown in the proof of [5, Theorem 1.1], it follows that $J_{1}=J_{G^{\prime}}$ where $G^{\prime}$ is obtained from $G$ by replacing the cliques $F_{t_{1}}, \ldots, F_{t_{q}}$ and $F_{r}$ by the clique on the vertex set $F_{r} \cup\left(\bigcup_{j=1}^{q} F_{t_{j}}\right)$. Also, $J_{2}=\left(x_{i}, y_{i}\right)+J_{G^{\prime \prime}}$ where $G^{\prime \prime}$ is the restriction of $G$ to the vertex set $[n] \backslash\{i\}$. 
We have $\operatorname{in}_{<}\left(J_{G}\right)=\operatorname{in}_{<}\left(J_{1} \cap J_{2}\right)$. By [1, Lemma 1.3], we have in $\operatorname{in}_{<}\left(J_{1} \cap\right.$ $\left.J_{2}\right)=\operatorname{in}_{<}\left(J_{1}\right) \cap \operatorname{in}_{<}\left(J_{2}\right)$ if and only if $\operatorname{in}_{<}\left(J_{1}+J_{2}\right)=\operatorname{in}_{<}\left(J_{1}\right)+\operatorname{in}_{<}\left(J_{2}\right)$. But $\operatorname{in}_{<}\left(J_{1}+J_{2}\right)=\operatorname{in}_{<}\left(J_{G^{\prime}}+\left(x_{i}, y_{i}\right)+J_{G^{\prime \prime}}\right)=\operatorname{in}_{<}\left(J_{G^{\prime}}+\left(x_{i}, y_{i}\right)\right)$. Hence, by Lemma 3.1 , we get in $\operatorname{in}_{<}\left(J_{1}+J_{2}\right)=\operatorname{in}_{<}\left(J_{G^{\prime}}\right)+\left(x_{i}, y_{i}\right)=\operatorname{in}_{<}\left(J_{1}\right)+\operatorname{in}_{<}\left(J_{2}\right)$. Therefore, we get $\operatorname{in}_{<}\left(J_{G}\right)=\operatorname{in}_{<}\left(J_{1}\right) \cap \operatorname{in}_{<}\left(J_{2}\right)$ and, consequently, we have the following exact sequence of $S$-modules

$$
0 \longrightarrow \frac{S}{\operatorname{in}_{<}\left(J_{G}\right)} \longrightarrow \frac{S}{\operatorname{in}_{<}\left(J_{1}\right)} \oplus \frac{S}{\operatorname{in}_{<}\left(J_{2}\right)} \longrightarrow \frac{S}{\operatorname{in}_{<}\left(J_{1}+J_{2}\right)} \longrightarrow 0 .
$$

By using again Lemma 3.1, we have in $\operatorname{in}_{<}\left(J_{2}\right)=\operatorname{in}_{<}\left(\left(x_{i}, y_{i}\right), J_{G^{\prime \prime}}\right)=\left(x_{i}, y_{i}\right)+$ in $_{<}\left(J_{G^{\prime \prime}}\right)$. Thus, we have actually the following exact sequence

$$
0 \longrightarrow \frac{S}{\operatorname{in}_{<}\left(J_{G}\right)} \longrightarrow \frac{S}{\operatorname{in}_{<}\left(J_{G^{\prime}}\right)} \oplus \frac{S}{\left(x_{i}, y_{i}\right)+\operatorname{in}_{<}\left(J_{G^{\prime \prime}}\right)} \longrightarrow \frac{S}{\left(x_{i}, y_{i}\right)+\operatorname{in}_{<}\left(J_{G^{\prime}}\right)} \longrightarrow 0 .
$$

Since $G^{\prime}$ inherits the properties of $G$ and has a smaller number of maximal cliques than $G$, it follows, by the inductive hypothesis, that

$$
\operatorname{depth}\left(S / J_{G^{\prime}}\right)=\operatorname{depth}\left(S / \operatorname{in}_{<}\left(J_{G^{\prime}}\right)\right)=n+1 .
$$

Let $S_{i}$ be the polynomial ring $S /\left(x_{i}, y_{i}\right)$. Then $S /\left(\left(x_{i}, y_{i}\right)+\operatorname{in}_{<}\left(J_{G^{\prime \prime}}\right)\right) \cong$ $S_{i} /$ in $_{<}\left(J_{G^{\prime \prime}}\right)$. Since $G^{\prime \prime}$ is a graph on $n-1$ vertices with $q+1$ connected components and satisfies our conditions, the inductive hypothesis implies that $\operatorname{depth} S /\left(\left(x_{i}, y_{i}\right)+\operatorname{in}_{<}\left(J_{G^{\prime \prime}}\right)\right)=n+q \geq n+1$. Hence,

$$
\operatorname{depth}\left(S / \operatorname{in}_{<}\left(J_{G^{\prime}}\right) \oplus S /\left(\left(x_{i}, y_{i}\right)+\operatorname{in}_{<}\left(J_{G^{\prime \prime}}\right)\right)\right)=n+1 .
$$

Next, we observe that $S /\left(\left(x_{i}, y_{i}\right)+\operatorname{in}_{<}\left(J_{G^{\prime}}\right)\right) \cong S_{i} / \operatorname{in}_{<}\left(J_{H}\right)$, where $H$ is obtained from $G^{\prime}$ by replacing the clique on the vertex set $F_{r} \cup\left(\bigcup_{j=1}^{q} F_{t_{j}}\right)$ by the clique on the vertex set $F_{r} \cup\left(\bigcup_{j=1}^{q} F_{t_{j}}\right) \backslash\{i\}$. Hence, by the inductive hypothesis, $\operatorname{depth}\left(S /\left(\left(x_{i}, y_{i}\right)+\mathrm{in}_{<}\left(J_{G^{\prime}}\right)\right)\right)=n$ since $H$ is connected and its vertex set has cardinality $n-1$. Hence, by applying the Depth lemma to exact sequence (1), we get

$$
\operatorname{depth} S / J_{G}=\operatorname{depth} S / \operatorname{in}_{<}\left(J_{G}\right)=n+1 .
$$

Definition 3.3. Let $\ell \geq 2$ be an integer. A $C_{\ell^{-}}$graph is a connected graph $G$ on the vertex set $[n]$ which consists of

(i) a sequence of maximal cliques $F_{1}, \ldots, F_{\ell}$ with $\operatorname{dim} F_{i} \geq 1$ for all $i$ such that $\left|F_{i} \cap F_{i+1}\right|=1$ for $1 \leq i \leq \ell-1$ and $F_{i} \cap F_{j}=\emptyset$ for any $i<j$ such that $j \neq i+1$, together with 
(ii) some additional edges of the form $F=\{j, k\}$ where $j$ is an intersection point of two consecutive cliques $F_{i}, F_{i+1}$ for some $1 \leq i \leq \ell-1$, and $k$ is a vertex of degree 1 .

In other words, $G$ is obtained from a graph $H$ with $\Delta(H)=\left\langle F_{1}, \ldots, F_{l}\right\rangle$ whose binomial edge ideal is Cohen-Macaulay (see [5, Theorem 3.1]) by attaching edges in the intersection points of the facets of $\Delta(H)$. Obviously, such a graph has the property that its longest induced path has length equal to $\ell$. In the case that $\operatorname{dim} F_{i}=1$ for $1 \leq i \leq \ell$, then $G$ is called a caterpillar graph. Figure 1 displayes a $C_{\ell}$-graph with $\ell=5$.

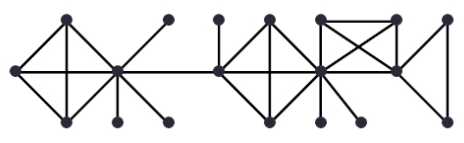

Figure 1: $C_{\ell \text {-graph }}$

We should also note that any $\mathcal{C}_{\ell^{-}}$graph is chordal and has the property that any two distinct maximal cliques intersect in at most one vertex. So that any $C_{\ell}$-graph is a connected block graph. But, obviously, there are block graphs which are not $C_{\ell^{-}}$graphs. Such an example is displayed in Figure 2.

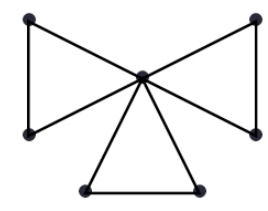

Figure 2: A block graph which is not a $C_{\ell^{-} \text {-graph }}$

Theorem 3.4. Let $G$ be a $\mathcal{C}_{\ell-g r a p h}$ on the vertex set $[n]$. Then

$$
\operatorname{reg}\left(S / J_{G}\right)=\operatorname{reg}\left(S / \operatorname{in}_{<}\left(J_{G}\right)\right)=\ell .
$$

Proof. Let $G$ consists of the sequence of maximal cliques $F_{1}, \ldots, F_{\ell}$ as in condition (i) in Definition 3.3 to which we add some edges as in condition (ii). So the maximal cliques of $G$ are $F_{1}, \ldots, F_{\ell}$ and all the additional whiskers. We proceed by induction on the number $r$ of maximal cliques of $G$. If $r=\ell$, then $G$ is a closed graph whose binomial edge ideal is Cohen-Macaulay, hence the statement holds by $\left[6\right.$, Theorem 3.2]. Let $r>\ell$ and let $F_{1}^{\prime}, \ldots, F_{r}^{\prime}$ be a leaf order on the facets of $\Delta(G)$. Obviously, we may choose a leaf order on $\Delta(G)$ 
such that $F_{r}^{\prime}=F_{\ell}$. With the same arguments and notation as in the proof of Theorem 3.2, we get the sequence (1).

We now observe that $G^{\prime}$ is a $\mathcal{C}_{\ell-1}$-graph, hence, by the inductive hypothesis,

$$
\operatorname{reg} \frac{S}{J_{G^{\prime}}}=\operatorname{reg} \frac{S}{\operatorname{in}_{<}\left(J_{G^{\prime}}\right)}=\ell-1 .
$$

The graph $G^{\prime \prime}$ has at most two non-trivial connected components. One of them, say $H_{1}$, is a $\mathcal{C}_{\ell^{\prime}}$-graph with $\ell^{\prime} \in\{\ell-2, \ell-1\}$. The other possible non-trivial component, say $H_{2}$, occurs if $\left|F_{\ell}\right| \geq 3$ and, in this case, $H_{2}$ is a clique of dimension $\left|F_{\ell}\right|-2 \geq 1$. By the inductive hypothesis, we obtain

$$
\operatorname{reg} \frac{S}{J_{G^{\prime \prime}}}=\operatorname{reg} \frac{S}{\operatorname{in}_{<}\left(J_{G^{\prime \prime}}\right)}=\operatorname{reg} \frac{S}{J_{H_{1}}}+\operatorname{reg} \frac{S}{J_{H_{2}}} \leq \ell-1+1=\ell .
$$

Relations (2) and (3) yield $\operatorname{reg}\left(S / \operatorname{in}_{<}\left(J_{G^{\prime}}\right) \oplus S /\left(\left(x_{i}, y_{i}\right)+\operatorname{in}_{<}\left(J_{G^{\prime \prime}}\right)\right)\right) \leq \ell$. From the exact sequence (1) we get

$\operatorname{reg}\left(\frac{S}{\operatorname{in}_{<}\left(J_{G}\right)}\right) \leq \max \left\{\operatorname{reg}\left(\frac{S}{\operatorname{in}_{<}\left(J_{G^{\prime}}\right)} \oplus \frac{S}{\left(x_{i}, y_{i}\right)+\operatorname{in}_{<}\left(J_{G^{\prime \prime}}\right)}\right), \operatorname{reg} \frac{S}{\operatorname{in}_{<}\left(J_{G^{\prime}}\right)}+1\right\} \leq \ell$.

By [7, Theorem 3.3.4], we know that $\operatorname{reg}\left(S / J_{G}\right) \leq \operatorname{reg}\left(S / \operatorname{in}_{<}\left(J_{G}\right)\right.$, and by [10, Theorem 1.1], we have $\operatorname{reg}\left(S / J_{G}\right) \geq \ell$. By using all these inequalities, we get the desired conclusion.

\section{Binomial edge ideals of caterpillar trees}

Matsuda and Murai showed in [10] that, for any connected graph $G$ on the vertex set $[n]$, we have $\ell \leq \operatorname{reg}\left(S / J_{G}\right) \leq n-1$, where $\ell$ denotes the length of the longest induced path of $G$, and conjectured that $\operatorname{reg}\left(S / J_{G}\right)=n-1$ if and only if $T$ is a line graph. Several recent papers are concerned with this conjecture; see, for example, [6], [13], and [14]. One may ask as well to characterize connected graphs $G$ whose longest induced path has length $\ell$ and $\operatorname{reg}\left(S / J_{G}\right)=\ell$. In this section, we answer this question for trees.

A caterpillar tree is a tree $T$ with the property that it contains a path $P$ such that any vertex of $T$ is either a vertex of $P$ or it is adjacent to a vertex of $P$. Clearly, any caterpillar tree is a $\mathcal{C}_{\ell}$-graph for some positive integer $\ell$.

Caterpillar trees were first studied by Harary and Schwenk [9]. These graphs have applications in chemistry and physics [4]. In Figure 3, an example of caterpillar tree is displayed. Note that any caterpillar tree is a narrow graph in the sense of Cox and Erskine [2]. Conversely, one may easily see that any narrow tree is a caterpillar tree. Moreover, as it was observed in [11], a tree is 


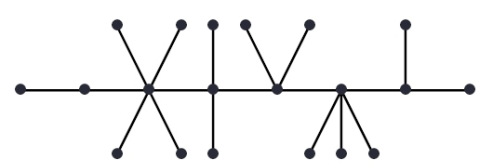

Figure 3: Caterpillar

a caterpillar graph if and only if it is weakly closed in the sense of definition given in [11].

In the next theorem we characterize the trees $T$ with $\operatorname{reg}\left(S / J_{T}\right)=\ell$ where $\ell$ is the length of the longest induced path of $T$.

Theorem 4.1. Let $T$ be a tree on the vertex set $[n]$ whose longest induced path $P$ has length $\ell$. Then $\operatorname{reg}\left(S / J_{T}\right)=\ell$ if and only if $T$ is caterpillar.

Proof. Let $T$ be a caterpillar tree whose longest induced path has length $\ell$. Then, by the definition of a caterpillar tree, it follows that $T$ is a $\mathcal{C}_{\ell^{-}}$graph. Hence, $\operatorname{reg}\left(S / J_{T}\right)=\ell$ by Theorem 3.4. Conversely, let $\operatorname{reg}\left(S / J_{T}\right)=\ell$ and assume that $T$ is not caterpillar. Then $T$ contains an induced subgraph $H$ with $\ell+3$ vertices as in Figure 4 .

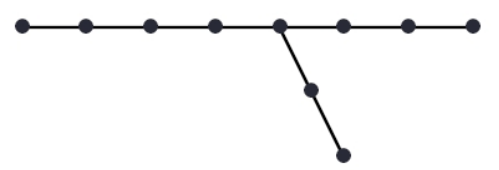

Figure 4: Induced graph $\mathrm{H}$

Then, by [15, Theorem 27], it follows that $\operatorname{reg}\left(S / J_{H}\right)=\ell+1$. Thus, since $\operatorname{reg}\left(S / J_{H}\right) \leq \operatorname{reg}\left(S / J_{G}\right)$ (see [10, Corollary 2.2]), it follows that $\operatorname{reg}\left(S / J_{G}\right) \geq$ $\ell+1$, contradiction to our hypothesis.

\section{References}

[1] A. Conca, Gorenstein ladder determinantal rings, J. London Math. Soc. 54(3) (1996), 453-474.

[2] D. A. Cox and A. Erskine, On closed graphs I, arXiv:1306.5149.

[3] A. Dokuyucu, Extremal Betti numbers of some classes of binomial edge ideals, to appear in Math. Reports. 
[4] S. El-Basil, Applications of caterpillar trees in chemistry and physics, J. Math. Chem. 1(2) (1987), 153-174.

[5] V. Ene, J. Herzog, T. Hibi, Cohen-Macaulay binomial edge ideals, Nagoya Math. J. 204 (2011), 57-68.

[6] V. Ene and A. Zarojanu, On the regularity of binomial edge ideals, Math. Nachr. 288(1) (2015), 19-24.

[7] J. Herzog and T. Hibi, Monomial Ideals, Graduate Texts in Mathematics 260, Springer, 2010.

[8] J. Herzog, T. Hibi, F. Hreinsdotir, T. Kahle, J. Rauh, Binomial edge ideals and conditional independence statements, Adv. Appl. Math. 45 (2010), $317-333$.

[9] F. Harary and A. J. Schwenk, The number of caterpillars, Discrete Math. 6(4), (1973), 359-365.

[10] K. Matsuda and S. Murai, Regularity bounds for binomial edge ideals, J. Commut. Algebra 5 (2013), 141-149.

[11] K. Matsuda, Weakly closed graphs and F-purity of binomial edge ideals, arXiv: 1209.4300 .

[12] M. Ohtani, Graphs and Ideals generated by some 2-minors, Commun. Algebra 39 (2011), no. 3, 905-917.

[13] S. Saeedi Madani and D. Kiani, Binomial edge ideals of graphs, Electron. J. Combin, 19 (2012), no. 2, \# P44.

[14] S. Saeedi Madani and D. Kiani, The regularity of binomial edge ideals of graphs, arXiv: 1310.6126.

[15] S. Zafar and Z. Zahid, On the Betti numbers of some classes of binomial edge ideals, Electron. J. Combin. 20 (2013) no. 4, \# P37.

Faryal CHAUDHRY,

Abdus Salam School of Mathematical Sciences,

GC University, Lahore

68-B, New Muslim Town, Lahore 54600, Pakistan.

Email: chaudhryfaryal@gmail.com 
Ahmet DOKUYUCU,

Faculty of Mathematics and Computer Science,

Ovidius University of Constanta,

Bd. Mamaia 124, 900527 Constanta, Romania, and Department of Information Technology,

Lumina-The University of South-East Europe,

Sos. Colentina nr. 64b, Bucharest, Romania.

Email: ahmet.dokuyucu@lumina.org

Rida IRFAN,

Abdus Salam School of Mathematical Sciences,

GC University, Lahore

68-B, New Muslim Town, Lahore 54600, Pakistan.

Email: ridairfan_88@yahoo.com 